\title{
Caracterización del Yacimiento a partir de los $d$ obtenidos en la toma de registros a Hueco Abie usando Redes Neuronales Artific
}

\author{
I \\ Jhan Harold Dsoric Sánchez.Ing. de Petróleos. USCD. \\ Efraín Pedraza Salazar. Ing. de Petróleas. USCD.
}

J] entrodela ingeniería de yacimientos petrolferos, existe la necesidad de caracterizar los yacimientos. Para esto se requiere obtener algunas rapiedades que son características del yacimiento como son la Porosidad y la Permeabilidad de la roca. Existen varias formas de obtener estas rropiedades; una de ellas es mediante pruebas delaboratoriousando muestras de corazones ylaotraes mediante registros de huecoabierto. Por el athcostode corazonamientode los pozos en laindustriadel petróleo se acostumbra tomar lamenor cantidad de corazones ytratar de encontrar lamejor trma de caracterizar el yacimiento mediante los registros de pozo a hueco abierto. Para realizar estas caracterizaciones se han definido algunos aroeptos que sirven de base paramejorar laderivaciónde lapermeabilidad ylaporasidad; incorporandolainfluenciadelasdistintas variables geológicas aecontrolanelflujode fluidos. Estas canceptos son:

Indicador de Zonade Fluja(FZ)

Indice de Calidad de Yacimiento(RLI)

Unidades Hidráulicasde Flujo(UHF)

Eneste artículo se planteala caracterizacín del yacimiento mediantel los registros a hueco abierto (usandalas Redes Neuranales Artificiales (RNA) nediantel software NEURDSQLUTILNS aplicando las topologías perceptron multicapa (MLP). y red generalizada hacia delante (GFF)), pertenecientea uncampodeterminadode Colombia. Las principales conclusiones y resultados son:

La RNA basada en latapología MLP tieneun coeficiente de correlación mas attopara la generaciónde porosidad, permeabilidad, FZl, RLD, UHF, que el generado por la RNA basada en la topología de conexióngeneralizada haciadelante, indicándome un mejor grado de exactitud para la MLP.

La sensibilidad de los datos de entradaparaladeterminacióndela porosidad, permeabilidad, FZl, RQl,yUHF son menores en las RNA's basadas en latopologiade conexióngeneralizada hacia delante que enla RNA basadaen la topología perceptronmulticapa.

Si l yacimienta es muy heterogéneo o si sus propiedades petrufísicas varían muchode un sector del yacimiento a otro, es conveniente ajustar la aplicación(reentrenarla RNA).

Se podría utilizar la misma aplicación para carrelacionar la infarmación de dos yacimientos, si laformación que se esta analizanda tienen litologías parecidas.

La importancia de este proyecto se basaenlareduccín de riesgos y costos a la hora de caracterizar el yacimientousando las Redes Neuronales Artificiales ya que estaaplicación genera datos confiables mediante registros tomados a hueco abierto correlacionando estos registros con los datos obtenidos basados en las muestras de un pozo corazonadode unyacimiento.

Las Redes Neuronales Artificiales sonaplicables paratemas especficos de análisisdedatas, interpretaciónderegistros depozo, modelamiertude llỵc multifasico en tubería, tratamientadedatos símmicos, caracterización de yacimientos naturalmenteffracturados, entre otros. 
Caracterización del yacimiento a partir de los datos obtenidos en la toma de registros a bueco abierto usando redes neuronales artificiales

Las Redes Neuronales Artificiales han sido usadas eficientemente en la solución de problemas donde es necesario correlacionar patrones, en la industria de los hidrocarburos las han utilizado en diferentes áreas de investigación.

Al tener como base las aplicaciones que se han realizado con las RNA en la industria de los hidrocarburos y los resultados obtenidos, se propone en este trabajo una solución alternativa a un problema planteado en la industria del petróleo, el cual es caracterizar un yacimiento de crudo basándose en las propiedades de porosidad y permeabilidad tomadas por registros a hueco abierto (Gamma Ray, SP, Resistividad y Registros de Porosidad). Para realizar estas caracterizaciones se han definido algunos conceptos como el FZI, RQI, UHF, que sirven de base para mejorar la derivación de la permeabilidad y la porosidad; incorporando la influencia de las distintas variables geológicas que controlan el flujo de fluidos.

- Indicador de Zona de Flujo (FZI)

- Índice de Calidad de Yacimiento (RQI)

s Unidades Hidráulicas de Flujo (UHF)

Dentro de la ingeniería de yacimientos petrolíferos, existe la necesidad de caracterizar los yacimientos. Para esto se requiere obtener algunas propiedades que son características del yacimiento como son la Porosidad y la Permeabilidad de la roca.

Existen varias formas de obtener estas propiedades; una de ellas es mediante pruebas de laboratorio usando muestras de corazones y la otra es mediante registros de hueco abierto. Por el alto costo de corazonamiento de los pozos en la industria del petróleo acostumbra tomar la menor cantidad de corazones y tratar encontrar mejor forma de caracterizar el yacimiento mediant los registros de pozo a hueco abierto.
En este trabajo se plantea la caracterización del yacimiento mediante los registros a hueco abierto (usando las Redes Neuronales Artificiales (RNA) mediante el software NEUROSOLUTIONS aplicando las topologías Perceptron Multicapa (MLP), y Red Generalizada hacia Adelante (GFF)), perteneciente a un campo determinado de Colombia.

La metodología planteada en el desarrollo de este proyecto es la siguiente:

1) Se recopila la información existente del campo (Registro a hueco abierto para cada uno de los pozos, incluyendo el pozo corazonado).

2) Se usan los datos obtenidos mediante los procedimientos de interpretación petrofísica (corazones) y se correlacionan con los registros a hueco abierto tomados al pozo corazonado mediante la profundidad real.

s) Se realiza filtro de los registros basándose en la diferencia entre el registro Caliper y el diámetro del hueco. Con el fin de reducir la probabilidad de inconsistencias en los resultados generados por las RNA's.

4) Se procede a escoger los registros que presenten mejor correlación con la variable a modelar. Para el caso de las unidades hidráulicas, modelamos las variables FZI y RQI. Estás variables se modelan mediante la permeabilidad y porosidad partiendo de los registros de mayor grado de correlación que en su orden son: SPc, GRe, Rt, RHOB.

5) Se Normalizan los registros a hueco abierto con el fin de reducir la probabilidad de análisis de registros. 
Metadalogia utilizada para la caracterizacián de un yacimienta mediante RNA's

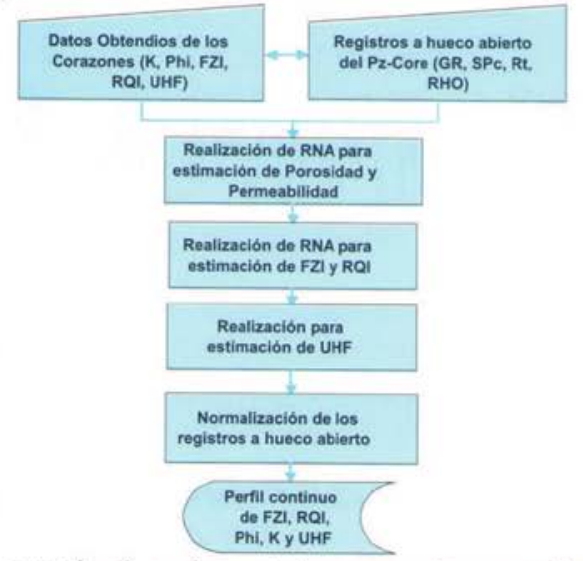

De acuerdo al cuadro anterior se puede generalizar para este estudio que la capacidad de flujo va disminuyendo a medida que aumenta la clasificación de la Unidad Hidráulica; siendo la Unidad Hidráulica
No.1, la de mejor capacidad de flujo y la Unidad No 5 , la de menor capacidad de flujo.

\section{Resultados}

Para analizar el comportamiento de la Red Neuronal desarrollada se le hicieron cuatro análisis básicos:

1. Se realizó un análisis de minimización del MSE durante el entrenamiento de la RNA.

2. Se realizó una validación o prueba de la RNA con la información obtenida de un pozo diferente al corazonado pero del mismo yacimiento.

3. Se evalúo la sensibilidad de los datos de entrada con respecto a cada uno de los datos generados por la RNA.

4. Se evalúo la influencia del dato de entrada sobre el dato generado por la RNA y se verifica que corresponda con la realidad.

Clasificación de las Unidades Hidráulicas de acuerdo a los valores promedio de FZI, Porosidad y Permeabilidad.

\begin{tabular}{cccc}
\hline Unidad Hidráulica & FZI promedio & $\begin{array}{c}\text { Porosidad media } \\
(\%)\end{array}$ & $\begin{array}{c}\text { Permeabilidad Media } \\
(\mathbf{m D})\end{array}$ \\
\hline $\mathbf{1}$ & 19.12 & 14.8 & 1128.332 \\
$\mathbf{2}$ & 8.284 & 10.8 & 49.482 \\
$\mathbf{3}$ & 4.19 & 9.6 & 10.461 \\
$\mathbf{4}$ & 1.838 & 9 & 1.933 \\
$\mathbf{5}$ & 0.874 & 11.1 & 1.139 \\
\hline
\end{tabular}

Fuente: Estudio de Daño a la formación - ICP. 2002.

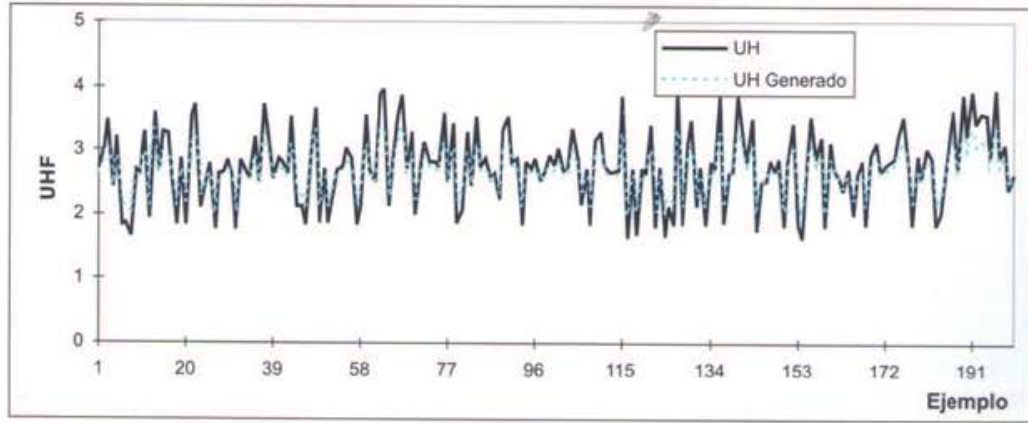

Comparación entre las Unidades Hidráulicas generadas por la RNA con topología MLP y las Unidades Hidráulicas obtenidas para el pozo en estudio. 
Caracterización del yacimiento a partir de los datos obtenidos en la toma de registros a hueco abierto usando redes neuronales artificiales

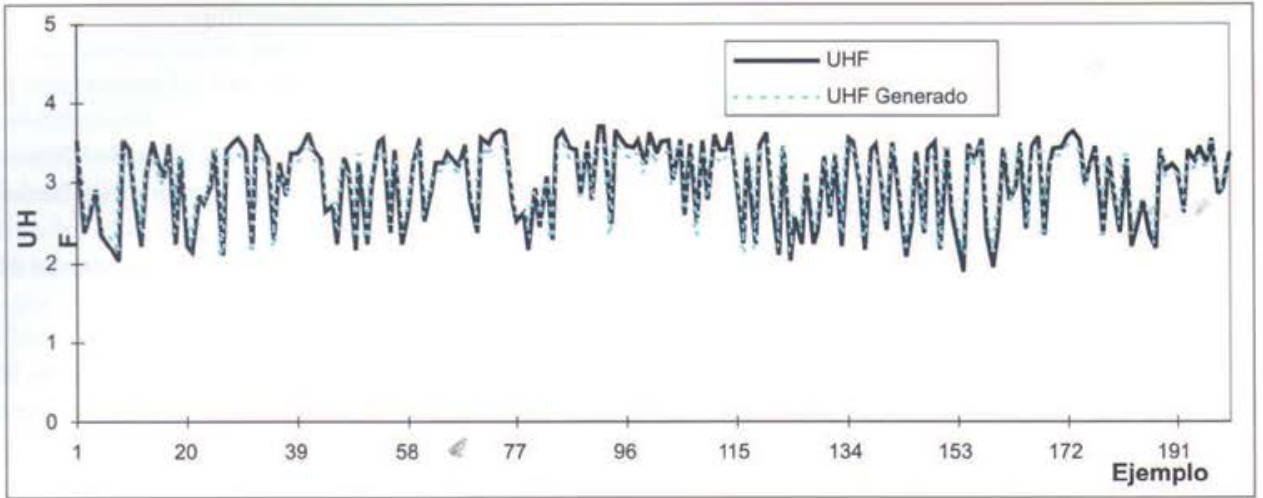

Comparación entre las Unidades Hidráulicas generadas por la RNA con topología GFF y las Unidades Hidráulicas obtenidas para el pozo en estudio.

\section{Conclusiones}

- Las lecturas del registro de densidad es el dato de entrada más sensible en las RNA's generadas, debido a que requiere un mayor grado de exactitud (cifras significativas) para garantizar la estabilidad de la aplicación generada.

- El uso de la validación cruzada fue considerada como una buena opción para mejorar el método de entrenamiento, ya que se reduce el MSE y la desviación estándar en cada una de las redes neuronales donde se utilizó.

- La RNA's basada en la topología de conexión generalizada hacia delante maneja de mejor forma las variaciones de las lecturas de los registros debido a las irregularidades presentes en la pared del pozo en la generación de los resultados (Presenta mayor estabilidad que la aplicación basada en la topología MLP).

s Si el yacimiento es muy heterogéneo o si sus propiedades petrofísicas varían mucho de un sector del yacimiento a otro, es conveniente ajustar la aplicación (Reentrenar la RNA).

- Se podría utilizar la misma aplicación para correlacionar la información de dos yacimientos, si la formación que se esta analizando tienen litologías parecidas.

\section{Recumendaciones}

s En el uso de Redes Neuronales para caracterizar un yacimiento, se recomienda generar RNA's que se entrenen con base a las características específicas de ese yacimiento.

c Los datos usados para el entrenamiento de las RNA's generadas se tomen de pozos que hayan sido corazonados porque son datos mas exactos y confiables.

- Se debe realizar filtro a las lecturas de los registros basándose en la diferencia de la lectura del Caliper y el diámetro del pozo, para garantizar la estabilidad y calidad de los resultados.

- El uso de las RNA es adecuado para la caracterización de yacimientos, debido a la tolerancia en las variaciones de los datos de entrada (especialmente si utilizamos la RNA de topología GFF), disminución de cálculos con formulas empíricas y uso de gráficas analíticas. s Para el entrenamiento de las redes neuronales se debe usar el método de validación cruzada solamente si tenemos más de cuatro patrones de datos de entrada, con el fin de generar una mejor correlación de datos. 


\section{Referencias Bibliagráficas}

1. ALI, J.K. Neural Networks a new tool for the petroleum industry. SPE 1994.

2. AMAEFULE, J.O., Altunbay, M., Tiab, D., Kersey,

D. G. and Keelan, D: K., Enhanced reservoir description using core and log data to identify hydraulic (flow) units and predict permeability in uncored intervals/wells. SPE 1993.

3. BALCH, R.S. Using artificial intelligence to correlate multiple seismic attributes to reservoir properties. SPE 1999.

4. COLL, C., Muggeeridge, A.H. and Jing, X.D. A new method to upscale waterflooding in heterogeneous reservoirs for a range of capillary and gravity effects. SPE 2000.

5. DELGADO, Alberto. Inteligencia artificial y minirobots. Editorial ecoe ediciones Santafé de Bogota D.C., julio 1998.

6. FREEMAN, James, A. Redes neuronales, algoritmos, aplicaciones y técnicas de programación. Editorial Wilmington, Delaware, EUA, Addison Wesley. Iberoamericana 1993.

7. GARCIA, G. y W: W. Whitman. Inversion of a lateral log using neural networks. SPE 1992.

8. KONAR, A. Artificial intelligence and soft computing behavior and cognitive modeling of the human brain. CRC press 2000.

9. KOUIDIER, A. y El Quahed. Application of artificial intelligence to characterize naturally fractured reservoirs. SPE 200 .

10.LIN, Y. and Cunninham, G.A. A new approach to fuzzy- neural system modeling. SPE 1995.

11. MARTINEZ, Victor, J, y Hilera, José, R. Redes Neuronales artificiales, fundamentos, modelos y aplicaciones. Editorial Wilmington, Delaware, EUA, Addison- Wesley. Iberoamericana 1995.

12. MOHAGHEGH, S. and Ameri, S. Artificial neural network as a valuable tool for petroleum engineers. SPE 1995.

13. PAVA, Pérez, Juan, Carlos. Redes neuronales en la enseñanza de las matemáticas. Editorial Universidad Surcolombiana, Neiva, 1999.
14. PEREZ, M.F, Rozo, M. A, Ulloa, R, Calvete, F. E. Herramienta Software para entrenar redes neuronales en aplicaciones de registros de pozos. Acipet 2003.

15.PRET, L. y Sánchez, S. Preedición del tipo de fluido de un yacimiento basado en datos PVT y de producción usando redes neuronales artificiales. Universidad Industrial de Santander. Escuela de Petróleos, Bucaramanga, 1997.

16.QUENES, A. Fractured Reservoir characterization and performance forecasting using geomechanics and artificial intelligence. SPE 1995.

17. QUINTERO, Eduardo, J. Characterization of naturally fractured reservoirs using artificial intelligence. SPE 2001.

18. RICHARDSON, QS and Weiss, W.W. Fractured Reservoir Characterization and performance forecasting using geomechanics and artificial intelligence. SPE 2000 .

19.SHACKLERFORD, J. Barry, Neural data structure programming with neurons. Hewlett Packard journal 1989.

20. SCHULUMBERGER, Ltd. Log interpretation principles/applications. Schlumberger educational services, Houston 1989.

21. SOCIETY OF EXPLORATION GEOPHYSICISTIS. Velocity-Permeability relations within hydraulic units. Geophysics 2002.

22. TABASSENKO, L. A guide to neural computing applications. Editorial Arnold 1998.

23. TOMSK, R. Hydraulic flow units resolve reservoir description. SPE 2001.

24. WINSTON, Patrick, Henry. Inteligencia artificial. Editorial Wilmington, Delaware, EUA, Addison-Wesley. Iberoamericana 1994.

25. ZELLOU, A.M, Quenes, A. Improved fractured reservoir characterization using neural networks, geomechanics and SD seismic. SPE 1995. 